\title{
BMJ Open Association between handgrip strength and the risk of new-onset metabolic syndrome: a population-based cohort study
}

\author{
Chao Shen, ${ }^{1}$ Jiangting Lu, ${ }^{1}$ Zhijie Xu, ${ }^{2}$ Yuanyuan $\mathrm{Xu},{ }^{2}$ Ying Yang (i) ${ }^{1}$
}

To cite: Shen C, Lu J, Xu Z, et al. Association between handgrip strength and the risk of new-onset metabolic syndrome: a population-based cohort study. BMJ Open 2020;10:e041384. doi:10.1136/ bmjopen-2020-041384

- Prepublication history for this paper is available online. To view these files, please visit the journal online (http://dx.doi. org/10.1136/bmjopen-2020041384).

Received 06 June 2020 Revised 26 August 2020 Accepted 28 August 2020
Check for updates

(c) Author(s) (or their employer(s)) 2020. Re-use permitted under CC BY-NC. No commercial re-use. See rights and permissions. Published by BMJ.

${ }^{1}$ Department of Cardiology, Zhejiang University School of Medicine Sir Run Run Shaw Hospital, Hangzhou, Zhejiang, China

${ }^{2}$ Department of General Practice, Zhejiang University School of Medicine Sir Run Run Shaw Hospital, Hangzhou, Zhejiang, China

Correspondence to

Dr Ying Yang; yylong@zju.edu.cn

\section{ABSTRACT}

Objectives A lower relative handgrip strength (HGS) may disrupt metabolic homeostasis and then lead to metabolic syndrome (MetS). There is a paucity of longitudinal studies to examine whether relative HGS at baseline is linked to incident MetS. Thus, the purpose of the present study was to explore the association between relative HGS and newonset MetS.

Design This is an observational and longitudinal research.

\section{SETTING}

A nationally representative sample of population in China. Participants A total of 3350 subjects without MetS were selected for analysis in the present study. Data are from the China Health and Retirement Longitudinal Study (2011-2015).

Outcome measures We calculated the relative HGS by dividing the HGS by body weight. Participants were divided into gender-specific quartiles. We estimated HRs for MetS and its components using Cox proportional hazard models according to the relative HGS categories.

Results After multiple adjustment, the risk of MetS increased with the lower quartile of relative HGS in both sexes. Using the highest quartile (Q4) as a reference, the HR for quartile Q3-1 was 1.49 (0.95, 2.34), 1.67 (1.08, $2.59)$ and $1.76(1.12,2.78)$, respectively, in men, and $1.14(0.82,1.58), 1.30(1.02,1.57)$ and $1.28(1.03,1.55)$, respectively, in women. Additionally, we observed that relative HGS was negatively or inversely associated with the risk of abdominal obesity in both sexes.

Conclusions The current study demonstrated that relative HGS was inversely and independently associated with an increased risk of MetS and abdominal obesity, suggesting a possible role of relative HGS as a useful and simple index for muscle strength in the prediction of occurrence of MetS.

\section{INTRODUCTION}

Metabolic syndrome (MetS) is defined as a clustering of multiple metabolic risk factors, including abdominal obesity, elevated blood pressure level, atherogenic dyslipidaemia and elevated fasting plasma glucose (FPG). The prevalence of MetS has attracted wide attention since it has become an adverse public health problem throughout the world

\section{Strengths and limitations of this study}

- This study was based on a nationally representative cohort (China Health and Retirement Longitudinal Study, CHARLS) in accordance with internationally acceptable standards.

- This was the first longitudinal study to estimate the association between relative handgrip strength (HGS) and the risk of metabolic syndrome (MetS) among a large representative population in China.

- Data were from CHARLS which lacked details on dietary habits, muscle mass, hormone levels and serum insulin.

- This study did not further explore the mechanism between relative HGS and MetS.

due to its close relationship with cardiovascular diseases and type 2 diabetes mellitus. ${ }^{1}$ Along with rapid economic development and changes in lifestyle and eating habits, the incidence of MetS has gradually increased over the past few decades in China. ${ }^{2}$ Recently, epidemiological research reported that the prevalence of MetS was 24.5\% among the Chinese population, and the morbidity rate was up to $32.4 \%$ among individuals older than 60 , whereas the total prevalence was $13.3 \%$ only 30 years ago. ${ }^{34}$ Therefore, exploring the risk factors and their interactions with MetS as a strategy to prevent MetS should be quickly implemented.

Skeletal muscle, which controls the movement of the body, is the fundamental reservoir of glucose and protein within the body. It provides gluconeogenic precursors to sustain metabolism. ${ }^{5}$ Inevitably, a reduction of skeletal muscle mass is an essential characteristic of ageing. ${ }^{6}$ The proportion of skeletal muscle declines by approximately $8 \%$ per decade after 40 years, and there is a steep decline by approximately $15 \%$ per decade after 60 years. ${ }^{7}$ The ability to perform activities of daily life is impaired by reduced muscle strength, 
Table 1 Baseline characteristics for both sexes according to development of metabolic syndrome

\begin{tabular}{|c|c|c|c|c|c|c|}
\hline & \multicolumn{3}{|l|}{ Men $(n=1845)$} & \multicolumn{3}{|c|}{ Women $(n=1505)$} \\
\hline & MetS $(n=217)$ & Non-MetS $(n=1628)$ & $P$ value & MetS $(n=362)$ & Non-MetS $(n=1143)$ & $P$ value \\
\hline Age, year & $59.2 \pm 9.1$ & $60.3 \pm 8.6$ & 0.043 & $58.1 \pm 8.8$ & $56.5 \pm 8.2$ & 0.005 \\
\hline Married & $198(91.2)$ & $1480(90.9)$ & 0.872 & $306(84.5)$ & 1017 (89.0) & 0.024 \\
\hline Region & & & $<0.001$ & & & 0.829 \\
\hline Rural & $167(77.0)$ & $1423(87.4)$ & & $319(88.1)$ & $1012(88.5)$ & \\
\hline Urban & $50(23.0)$ & 205 (12.6) & & 43 (11.9) & $131(11.5)$ & \\
\hline Education & & & 0.027 & & & 0.310 \\
\hline Illiterate & 27 (12.4) & 217 (13.3) & & $87(24.0)$ & $242(21.2)$ & \\
\hline Primary & $92(42.4)$ & $826(50.7)$ & & $151(41.7)$ & $463(40.5)$ & \\
\hline High & $98(45.2)$ & $584(35.9)$ & & $124(34.3)$ & 438 (38.3) & \\
\hline Current smoker & 38 (17.5) & $227(13.9)$ & $<0.001$ & $4(1.1)$ & $14(1.2)$ & 0.481 \\
\hline Alcohol intake & $16(7.4)$ & $193(11.9)$ & $<0.001$ & $16(4.4)$ & $62(5.4)$ & 0.734 \\
\hline \multicolumn{7}{|l|}{ History of disease } \\
\hline Hypertension & $548(29.7)$ & $452(27.8)$ & $<0.001$ & $120(33.1)$ & 212 (18.5) & $<0.001$ \\
\hline Hyperlipidaemia & $374(20.3)$ & 309 (19.0) & $<0.001$ & $92(25.4)$ & $174(15.2)$ & $<0.001$ \\
\hline Diabetes & $168(9.1)$ & $146(9.0)$ & 0.556 & $24(6.6)$ & $60(5.2)$ & 0.327 \\
\hline Handgrip strength, kg & $39.1 \pm 8.9$ & $37.4 \pm 8.8$ & 0.004 & $26.4 \pm 6.5$ & $26.4 \pm 6.6$ & 0.778 \\
\hline Height, cm & $164.7 \pm 6.2$ & $162.9 \pm 7.8$ & $<0.001$ & $152.6 \pm 10.4$ & $152.7 \pm 7.2$ & 0.640 \\
\hline Weight, kg & $64.6 \pm 8.9$ & $57.5 \pm 8.5$ & $<0.001$ & $56.0 \pm 9.2$ & $52.1 \pm 9.0$ & $<0.001$ \\
\hline $\mathrm{BMI}, \mathrm{kg} / \mathrm{m}^{2}$ & $23.7 \pm 2.6$ & $21.6 \pm 2.6$ & $<0.001$ & $23.8 \pm 3.3$ & $22.2 \pm 3.2$ & $<0.001$ \\
\hline $\mathrm{WC}, \mathrm{cm}$ & $85.3 \pm 13.1$ & $80.0 \pm 9.6$ & $<0.001$ & $83.3 \pm 12.2$ & $78.5 \pm 12.4$ & $<0.001$ \\
\hline SBP, $\mathrm{mm} \mathrm{Hg}$ & $132.1 \pm 20.9$ & $125.4 \pm 18.7$ & $<0.001$ & $125.6 \pm 24.6$ & $120.3 \pm 17.6$ & $<0.001$ \\
\hline DBP, $\mathrm{mm} \mathrm{Hg}$ & $77.6 \pm 12.6$ & $73.3 \pm 11.4$ & $<0.001$ & $73.7 \pm 10.7$ & $70.7 \pm 10.2$ & $<0.001$ \\
\hline $\mathrm{TC}, \mathrm{mg} / \mathrm{dL}$ & $192.0 \pm 34.6$ & $184.4 \pm 35.3$ & 0.002 & $200.6 \pm 38.0$ & $193.0 \pm 34.3$ & $<0.001$ \\
\hline $\mathrm{TG}, \mathrm{mg} / \mathrm{dL}$ & $113.5 \pm 56.6$ & $92.2 \pm 44.9$ & $<0.001$ & $104.5 \pm 38.6$ & $91.7 \pm 36.3$ & $<0.001$ \\
\hline LDL-C, mg/dL & $120.3 \pm 32.4$ & $111.3 \pm 31.8$ & $<0.001$ & $127.9 \pm 33.1$ & $117.3 \pm 29.7$ & $<0.001$ \\
\hline $\mathrm{HDL}-\mathrm{C}, \mathrm{mg} / \mathrm{dL}$ & $49.6 \pm 13.0$ & $56.2 \pm 15.6$ & $<0.001$ & $54.9 \pm 12.0$ & $60.0 \pm 13.2$ & $<0.001$ \\
\hline $\mathrm{FPG}, \mathrm{mg} / \mathrm{dL}$ & $104.5 \pm 26.4$ & $103.6 \pm 26.4$ & 0.594 & $102.2 \pm 25.1$ & $99.6 \pm 21.8$ & 0.071 \\
\hline $\mathrm{HbA} 1 \mathrm{c}, \%$ & $5.1 \pm 1.0$ & $5.1 \pm 0.7$ & 0.338 & $5.2 \pm 0.7$ & $5.1 \pm 0.8$ & $<0.001$ \\
\hline
\end{tabular}

Values are presented as mean \pm SD, number (\%).

BMI, body mass index; DBP, diastolic blood pressure; FPG, fasting plasma glucose; HbA1c, glycated haemoglobin; HDL-C, high-density lipoprotein cholesterol; LDL-C, low-density lipoprotein cholesterol; MetS, metabolic syndrome; SBP, systolic blood pressure; TC, total cholesterol; TG, triglyceride; WC, waist circumference.

and it renders the elderly vulnerable to the serious consequences of disability and multiple complications. ${ }^{8}$

Relative handgrip strength (HGS) has been considered to be a simple, reliable and convenient anthropometric index to assess the strength of skeletal muscle, and it is also a powerful predictor of sarcopenia. ${ }^{9}$ Compared with other HGS measurements, such as absolute HGS and dominant HGS, relative HGS is calculated by dividing the HGS by body weight thereby minimising the interference of body size and possible conflicting consequences. ${ }^{10}$ Although there is no standard HGS indicator, emerging evidence suggests that muscular fitness and functional decline, measured by relative HGS, are associated with cardiovascular diseases, mobility and all-cause mortality. ${ }^{11}{ }^{12}$ Additionally, it is commonly accepted that relative HGS is closely associated with the metabolic profile, including total cholesterol (TC), total triglyceride (TG), high-density lipoprotein cholesterol (HDL-C) and FPG. ${ }^{13-15}$ Based on the findings of prior studies, we can hypothesise that a decline of relative HGS may be a valuable indicator to predict the onset of MetS.

To best of our knowledge, several cross-sectional observations have reported that relative HGS has been associated with the prevalence of MetS. ${ }^{13}{ }^{15}$ However, it has not been determined whether and to what extent the longitudinal effects of relative HGS are associated with the incidence of MetS in China. Thus, in this study, we sought to dissect the longitudinal effects on the development of 
Table 2 Correlations between relative handgrip strength and metabolic parameters according to both sexes

\begin{tabular}{|c|c|c|c|c|}
\hline & \multicolumn{2}{|c|}{ Men $(n=1845)$} & \multicolumn{2}{|c|}{ Women $(n=1505)$} \\
\hline & $r$ & $P$ value & $r$ & $P$ value \\
\hline WC, $\mathrm{cm}$ & -0.188 & $<0.001$ & -0.251 & $<0.001$ \\
\hline SBP, mm Hg & -0.010 & 0.663 & -0.020 & 0.446 \\
\hline DBP, $\mathrm{mm} \mathrm{Hg}$ & -0.030 & 0.174 & -0.069 & 0.008 \\
\hline $\mathrm{TC}, \mathrm{mg} / \mathrm{dL}$ & -0.007 & 0.750 & -0.040 & 0.121 \\
\hline $\mathrm{TG}, \mathrm{mg} / \mathrm{dL}$ & -0.051 & 0.023 & -0.038 & 0.142 \\
\hline LDL-C, mg/dL & -0.049 & 0.028 & -0.033 & 0.199 \\
\hline $\mathrm{HDL}-\mathrm{C}, \mathrm{mg} / \mathrm{dL}$ & 0.112 & $<0.001$ & 0.023 & 0.363 \\
\hline $\mathrm{FPG}, \mathrm{mg} / \mathrm{dL}$ & -0.030 & 0.630 & -0.020 & 0.432 \\
\hline $\mathrm{HbA} 1 \mathrm{c}, \%$ & -0.032 & 0.153 & -0.054 & 0.037 \\
\hline
\end{tabular}

Pearson's correlation coefficient adjusted for age.

$\mathrm{DBP}$, diastolic blood pressure; FPG, fasting plasma glucose; $\mathrm{HbA1c}$, glycated haemoglobin; HDL-C, high-density lipoprotein cholesterol; LDL-C, low-density lipoprotein cholesterol; SBP, systolic blood pressure; TC, total cholesterol; TG, triglyceride; WC, waist circumference.

MetS across relative HGS quartiles and further analysed the association between separate MetS components and relative HGS in both men and women.

\section{METHODS}

\section{Study population}

Data for the present study came from the China Health and Retirement Longitudinal Study (CHARLS) from 2011 to 2015. The CHARLS is an ongoing longitudinal and representative national survey of middle-aged and older residents in China. This database contains highquality scientific information. It allows researchers to assess the prevalence of chronic diseases that reflect the current rapid ageing issues in China. More than 25000 residents from 450 villages/resident committees and 150 counties across 28 provinces were chosen randomly by the Charles-CIS software. All participants were interviewed face-to-face to gather information regarding demographic variables, lifestyle and health status, and they also underwent physical examinations by the trained investigators. Detailed information on the study design, sampling procedures and data analysis is publicly available. ${ }^{16}$

A total of 6341 participants had laboratory biomarkers evaluated twice, in 2011 and 2015. We excluded 2553 participants under the criteria of having MetS at baseline. Next, we also excluded 438 participants under 45 years old or missing anthropometric indicators. Finally, 3350 participants were included for analysis in our study. The data collection was approved by the Institutional Review Board (IRB) of Peking University (IRB 00001052-11015).

\section{Data collection}

We used a standardised and structural questionnaire to collect sociodemographic characteristics, such as age, sex, marital status (married or not), region (rural, urban), education (illiterate, primary school level, high school level), current smoker status, alcohol intake and a personal history of diseases (hypertension, hyperlipidaemia, diabetes).

Muscle strength was measured via HGS, using a handheld dynamometer (WCS-100, Nantong, China). With the guidance and demonstration by the trained technicians, participants in a standing position held the dynamometer at a right angle and squeezed the handles as hard as possible. The process of measurement was repeated two times with each hand alternately at an interval of at least 30 s. All measured values were recorded, and the maximum value was adopted. Considering the confounding effect of body weight in regards to muscle strength, we calculated the relative HGS by dividing the HGS by body weight in order to normalise the indicator. ${ }^{17-20}$

Anthropometric characteristics, including height, weight and waist circumference (WC), were measured using standardised protocols with the individuals wearing light clothing and no footwear. WC was measured at the midpoint between the last rib cage and iliac crest. Weight in kilograms was divided by the height in metres squared to calculate the value of body mass index (BMI) $(\mathrm{kg} /$ $\mathrm{m}^{2}$ ). The blood pressure of all individuals was measured three times by trained technicians. The participants sat quietly for at least $5 \mathrm{~min}$ before the measurements, and the average blood pressure was taken as the final value for analysis.

Venous blood samples were collected in the morning after overnight fasting, then immediately stored frozen at $-20^{\circ} \mathrm{C}$, and finally sent to the China Medical University laboratory. TC, TG, low-density lipoprotein cholesterol (LDL-C), HDL-C and FPG were measured using enzymatic colorimetric tests. Glycated haemoglobin (HbAlc\%) was analysed using high performance liquid chromatography. All procedures were completed by professional staff following standard testing methods.

Participants were defined as having MetS if they presented with three or more of the following criteria ${ }^{1}$ : (1) abdominal obesity, WC $\geq 90 \mathrm{~cm}$ in men and $\geq 80$ for women; (2) systolic blood pressure (SBP) $\geq 130 \mathrm{~mm} \mathrm{Hg}$ or diastolic blood pressure (DBP) $\geq 85 \mathrm{~mm} \mathrm{Hg}$ or taking antihypertensive medications; (3) $\mathrm{TG} \geq 150 \mathrm{mg} / \mathrm{dL}$ or under current drug treatment for high TG; (4) HDL-C $<40 \mathrm{mg}$ / $\mathrm{dL}$ in men, $<50 \mathrm{mg} / \mathrm{dL}$ in women or under current drug treatment for low HDL-C; (5) FPG $\geq 100 \mathrm{mg} / \mathrm{dL}$ or under current antihyperglycemic treatment.

\section{Patient and public involvement}

No patients were involved in this study.

\section{Statistical analysis}

We summarised the participants' sociodemographic, anthropometric and biomarker characteristics in the data analysis and analysed statistical differences according to whether MetS developed. Continuous variables were expressed as means and SD, while categorical variables 
were reported as frequencies and percentages. Student's t-tests and $\chi^{2}$ tests were used to compare the differences between groups that did or did not develop MetS.

Considering the potential bias effect that exists in the evaluation of HGS, we used relative HGS to estimate the HGS based on the standard assumptions about morphological effects. ${ }^{21}$ Subsequently, we used Pearson's correlation to examine the association between relative HGS and a series of metabolic parameters in men and women. The samples were divided into four categories (Q1-Q4) by quartile in terms of relative HGS. Simultaneously, we used multivariate Cox proportional hazards analysis to compare the HRs and 95\% CIs for each quartile, using the highest quartile as the reference group to assess the longitudinal effect of a lower relative HGS on the incidence of MetS.

The initial model was adjusted for age (Model 1). We additionally adjusted for married status, region, education, current smoker, alcohol consumption, hypertension, hyperlipidaemia and diabetes based on Model 1 (Model 2) and then we adjusted for WC based on Model 2 (Model 3) to analyse the associations between relative HGS and the development of MetS and its components. We calculated the $\mathrm{p}$ for trend to estimate the linear trend in the multivariate Cox proportional hazards analysis by entering the median value of each category of relative HGS as a continuous variable. Two-tailed $p<0.05$ was defined as statistically significant. All statistical analysis was performed using the statistical package SPSS V.24.0 (IBM) and R V.3.0.4 (R Foundation, Vienna, Austria; http://www.R-project.org/).

\section{RESULTS}

Table 1 presents the detailed baseline characteristic of the study participants according to the incidence of MetS. A total of 3350 subjects (1845 were men and 1505 were women) were selected for the present study. At baseline, the men who did develop MetS were 59.2 \pm 9.1 years of age and had a value of $39.1 \pm 8.9 \mathrm{~kg}$ for HGS, and those who did not develop MetS were $60.3 \pm 8.6$ years of age and had a value of $37.4 \pm 8.8 \mathrm{~kg}$. At baseline, the women who did develop MetS were 58.1 \pm 8.8 years of age and had a value of $26.4 \pm 6.5 \mathrm{~kg}$ for HGS, and those who did not develop MetS were $56.5 \pm 8.2$ years of age and had a value of $26.3 \pm 6.6 \mathrm{~kg}$.

Compared with participants who did not develop MetS, participants who did had significantly higher weight, BMI, WC, SBP, DBP, TC, TG, LDL-C, hypertension and hyperlipidaemia in both sexes. However, there were evident differences between the MetS group and the not-MetS group for region, education, current smoker, alcohol consumption only in men, and for married status, $\mathrm{HbA1c} \%$ only in women. There were no significant differences in FPG or a history of diabetes.

Table 2 displays the correlation between relative HGS and metabolic parameters. In men, relative HGS was negatively correlated with WC, TG and LDL-C, and positively with HDL-C. In women, relative HGS was negatively associated with WC, DBP and $\mathrm{HbAlc} \%$. Interestingly, WC was negatively associated with relative HGS in both men and women $(r=-0.188, p<0.001$ in men; $r=-0.251, p<0.001$ in women).

During the period of 13343.1 person-years (median follow-up 4.0 years), a total of 579 participants were diagnosed with MetS. Tables 3 and 4 present the HRs and 95\% CIs for developing MetS across the relative HGS categories in men and women, respectively. After adjustments for multiple confounders, including age, marital status, region, education, current smoker, alcohol consumption and history of diseases (hypertension, hyperlipidaemia, diabetes), using the highest quartile as the reference, the risk of MetS increased in the lower quartile of relative HGS in both sexes ( $\mathrm{p}$ for trend $<0.001$ ). The HRs for quartile Q3-1 were $1.49(0.95,2.34), 1.67(1.08,2.59)$ and $1.76(1.12,2.78)$, respectively, in men, and $1.14(0.82$, $1.58), 1.30(1.02,1.57)$ and $1.28(1.03,1.55)$, respectively, in women. Subsequently, we progressively analysed the association between relative HGS and metabolic risk factors. We only observed that a lower relative HGS was associated with a greater risk of abdominal obesity in both sexes. Using the highest quartile as the reference, the HRs for quartile Q3-1 were $1.50(1.04,2.16), 1.56(1.09,2.24)$ and $1.80(1.25,2.59)$, respectively, in men, and 1.24 (1.01, $1.52), 1.31(1.14,1.70)$ and $1.29(1.05,1.59)$, respectively, in women.

\section{DISCUSSION}

In this national cohort study, the significant association between relative HGS and the incidence of MetS was confirmed, and this association was more apparent in men than in women. The findings of our study highlight the pronounced effect of relative HGS on MetS. These results will support efforts to probe the mechanisms of MetS more accurately and to establish more appropriate strategies for the prevention of MetS.

A number of epidemiological studies have reported a similar relationship between relative HGS and MetS. Wu et $a l$ suggested that relative HGS is inversely related to MetS in a survey of 17703 individuals aged more than 40 years in Tianjin. ${ }^{18}$ A previous cross-sectional study reported that decreased relative HGS was closely correlated with disturbances of the metabolic profile and metabolic diseases across five provinces in China. ${ }^{19}$ The results of Yang et al also support our conclusion and emphasise the need to pay more attention to muscular strength in the elderly. ${ }^{22}$ Churilla et al reported that increased mean combined relative HGS may be inversely associated with the prevalence of MetS or its separate components using 20112014 National Health and Nutrition Examination Survey (NHANES) data. ${ }^{15}$ Sensitivity and specificity of relative HGS in predicting the MetS had been evaluated in recent studies, and the cut-off values of HGS per body weight were close in men and women, respectively. ${ }^{1823}$ However, there were no longitudinal studies to establish whether a 
Table 3 Hazard ratios for association between handgrip strength and metabolic syndrome and its components in men

\begin{tabular}{|c|c|c|c|c|c|}
\hline & \multicolumn{5}{|c|}{ Handgrip strength per body weight (kg/kg) } \\
\hline & \multirow{2}{*}{$\begin{array}{l}\text { Quartile 4 } \\
(0.73-1.17, n=461)\end{array}$} & \multirow{2}{*}{$\begin{array}{l}\text { Quartile 3 } \\
(0.65-0.72, n=461)\end{array}$} & \multirow{2}{*}{$\begin{array}{l}\text { Quartile 2 } \\
(0.57-0.64, n=461)\end{array}$} & \multirow{2}{*}{$\begin{array}{l}\text { Quartile } 1 \\
(0.15-0.56, n=462)\end{array}$} & \multirow[b]{2}{*}{ P for trenc } \\
\hline & & & & & \\
\hline Person-years & 1838.8 & 1834.4 & 1836.3 & 1837.1 & \\
\hline New-onset MetS & $30(6.5)$ & $50(10.8)$ & $66(14.3)$ & $68(14.8)$ & \\
\hline Model 1 & 1 (reference) & $1.77(1.13,2.73)$ & $2.19(1.43,3.33)$ & $2.51(1.63,3.84)$ & $<0.001$ \\
\hline Model 2 & 1 (reference) & $1.67(1.06,2.64)$ & $2.13(1.38,3.28)$ & $2.36(1.51,3.69)$ & $<0.001$ \\
\hline Model 3 & 1 (reference) & $1.49(0.95,2.34)$ & $1.67(1.08,2.59)$ & $1.76(1.12,2.78)$ & $<0.001$ \\
\hline \multicolumn{6}{|l|}{ MetS components } \\
\hline Abdominal obesity & $48(10.4)$ & $82(17.7)$ & $108(23.4)$ & $130(28.2)$ & \\
\hline Model 1 & 1 (reference) & $2.07(1.45,2.96)$ & $2.60(1.84,3.66)$ & $3.63(2.58,5.10)$ & $<0.001$ \\
\hline Model 2 & 1 (reference) & $1.96(1.37,2.83)$ & $2.45(1.73,3.27)$ & $3.32(2.34,4.71)$ & $<0.001$ \\
\hline Model 3 & 1 (reference) & $1.50(1.04,2.16)$ & $1.56(1.09,2.24)$ & $1.80(1.25,2.59)$ & $<0.001$ \\
\hline Elevated TG & 78 (16.9) & 78 (16.9) & 95 (20.6) & $86(18.7)$ & \\
\hline Model 1 & 1 (reference) & $1.19(0.87,1.63)$ & $1.38(1.02,1.87)$ & $1.43(1.04,1.96)$ & 0.006 \\
\hline Model 2 & 1 (reference) & $1.16(0.84,1.60)$ & $1.38(1.01,1.88)$ & $1.39(1.00,1.93)$ & 0.006 \\
\hline Model 3 & 1 (reference) & $1.10(0.80,1.52)$ & $1.29(0.94,1.76)$ & $1.31(0.94,1.82)$ & 0.024 \\
\hline Decreased HDL & $48(10.4)$ & 55 (11.9) & $60(13.0)$ & $63(13.7)$ & \\
\hline Model 1 & 1 (reference) & $1.29(0.87,1.90)$ & $1.30(0.89,1.91)$ & $1.47(1.00,2.17)$ & 0.009 \\
\hline Model 2 & 1 (reference) & $1.10(0.74,1.65)$ & $1.19(0.80,1.76)$ & $1.29(0.86,1.92)$ & 0.02 \\
\hline Model 3 & 1 (reference) & $1.08(0.73,1.61)$ & $1.15(0.78,1.71)$ & $1.22(0.81,1.83)$ & 0.054 \\
\hline High blood pressure & $157(34.0)$ & 195 (42.2) & $228(49.5)$ & 199 (43.2) & \\
\hline Model 1 & 1 (reference) & $1.28(1.04,1.58)$ & $1.35(1.10,1.66)$ & $1.15(0.93,1.43)$ & 0.195 \\
\hline Model 2 & 1 (reference) & $1.28(1.03,1.59)$ & $1.31(1.05,1.62)$ & $1.11(0.89,1.39)$ & 0.28 \\
\hline Model 3 & 1 (reference) & $1.26(1.01,1.56)$ & $1.27(1.03,1.57)$ & $1.06(0.85,1.33)$ & 0.551 \\
\hline Elevated FPG & $128(27.8)$ & $132(28.6)$ & $143(31.0)$ & 151 (32.8) & \\
\hline Model 1 & 1 (reference) & $1.09(0.86,1.40)$ & $1.10(0.86,1.40)$ & $1.16(0.91,1.48)$ & 0.044 \\
\hline Model 2 & 1 (reference) & $1.10(0.85,1.42)$ & $1.10(0.85,1.41)$ & $1.17(0.91,1.52)$ & 0.038 \\
\hline Model 3 & 1 (reference) & $1.09(0.85,1.39)$ & $1.06(0.83,1.36)$ & $1.11(0.86,1.43)$ & 0.103 \\
\hline
\end{tabular}

Tests for trend were conducted using multivariate Cox proportional hazards models. Values are presented as number (\%) or $\mathrm{HR}(95 \% \mathrm{Cl})$. Model 1: adjusted for age. Model 2: adjusted for marital status, region, education, current smoker, alcohol consumption, hypertension, hyperlipidaemia, diabetes based on Model 1. Model 3: adjusted for WC based on Model 2.

FPG, fasting plasma glucose; HDL-C, high-density lipoprotein cholesterol; MetS, metabolic syndrome; TG, triglyceride; WC, waist circumstance.

low relative HGS increased the risk of MetS among a large representative population in China, to best of our knowledge, the present study has filled this gap.

In this study, we demonstrated that a low relative HGS increased the risk of MetS, in turn, this phenomenon may be mainly attributed to a higher risk of abdominal obesity. WC is an adequate parameter to reflect the degree of ectopic adipose tissue accumulation. Keevil et al reported that HGS decreased by $3.56 \mathrm{~kg}$ in men and $1.00 \mathrm{~kg}$ in women per $10 \mathrm{~cm}$ increase in WC. ${ }^{24}$ However, recently, a study reported that abdominal obesity could accelerate the loss of muscle strength only in men. ${ }^{25} \mathrm{~A}$ previous study showed that increased relative HGS could ameliorate the negative effect of abdominal obesity on functional independence and performing activities of daily life among older adults. ${ }^{26}$

One explanation for these findings might involve partial pathophysiological mechanisms. Insulin resistance and intramuscular fat accumulation could be considered as contributing factors to age-related declines in muscle strength. ${ }^{27}$ Additionally, excessive intramuscular fat accumulation in skeletal muscles leads to further insulin resistance, which is the most widely accepted hypothesis to explain the development of MetS. ${ }^{29} 30$ On the other hand, decreased muscle strength may participate in changes in released inflammatory markers. Several researchers have observed that lower HGS is related to higher levels of inflammatory markers, such as interleukin (IL)-6 and 
Table 4 Hazard ratios for association between handgrip strength and metabolic syndrome and its components in women

\begin{tabular}{|c|c|c|c|c|c|}
\hline & \multicolumn{5}{|c|}{ Handgrip strength per body weight (kg/kg) } \\
\hline & \multirow{2}{*}{$\begin{array}{l}\text { Quartile } 4 \\
(0.59-1.00, n=376)\end{array}$} & \multirow{2}{*}{$\begin{array}{l}\text { Quartile 3 } \\
(0.51-0.58, n=375)\end{array}$} & \multirow{2}{*}{$\begin{array}{l}\text { Quartile 2 } \\
(0.43-0.50, n=377)\end{array}$} & \multirow{2}{*}{$\begin{array}{l}\text { Quartile } 1 \\
(0.15-0.42, n=377)\end{array}$} & \multirow[b]{2}{*}{ P for trenc } \\
\hline & & & & & \\
\hline Person-years & 1499.3 & 1493.5 & 1499.6 & 1504.1 & \\
\hline New-onset MetS & $69(18.4)$ & $80(21.3)$ & $100(26.5)$ & $113(30.0)$ & \\
\hline Model 1 & 1 (reference) & $1.19(0.86,1.64)$ & $1.50(1.10,2.04)$ & $1.49(1.10,2.02)$ & $<0.001$ \\
\hline Model 2 & 1 (reference) & $1.20(0.85,1.67)$ & $1.58(1.15,2.18)$ & $1.56(1.14,2.13)$ & $<0.001$ \\
\hline Model 3 & 1 (reference) & $1.14(0.82,1.58)$ & $1.30(1.02,1.57)$ & $1.28(1.03,1.55)$ & $<0.001$ \\
\hline \multicolumn{6}{|l|}{ MetS components } \\
\hline Abdominal obesity & $165(43.9)$ & $225(60.0)$ & $245(65.0)$ & $276(73.2)$ & \\
\hline Model 1 & 1 (reference) & $1.40(1.14,1.71)$ & $1.53(1.26,1.87)$ & $1.64(1.35,1.99)$ & $<0.001$ \\
\hline Model 2 & 1 (reference) & $1.42(1.15,1.75)$ & $1.62(1.32,1.99)$ & $1.68(1.37,2.06)$ & $<0.001$ \\
\hline Model 3 & 1 (reference) & $1.24(1.01,1.52)$ & $1.31(1.14,1.70)$ & $1.29(1.05,1.59)$ & $<0.001$ \\
\hline Elevated TG & 73 (19.4) & $87(23.2)$ & $95(25.2)$ & $87(23.1)$ & \\
\hline Model 1 & 1 (reference) & $1.22(0.90,1.67)$ & $1.35(0.99,1.83)$ & $1.16(0.85,1.59)$ & 0.186 \\
\hline Model 2 & 1 (reference) & $1.17(0.85,1.61)$ & $1.38(1.01,1.89)$ & $1.08(0.78,1.50)$ & 0.388 \\
\hline Model 3 & 1 (reference) & $1.13(0.82,1.55)$ & $1.28(0.94,1.74)$ & $1.00(0.72,1.38)$ & 0.660 \\
\hline Decreased HDL & $120(31.9)$ & $98(26.1)$ & $110(29.2)$ & 129 (34.2) & \\
\hline Model 1 & 1 (reference) & $0.84(0.64,1.10)$ & $0.95(0.73,1.23)$ & $1.04(0.81,1.34)$ & 0.711 \\
\hline Model 2 & 1 (reference) & $0.83(0.63,1.10)$ & $0.97(0.74,1.28)$ & $1.07(0.82,1.39)$ & 0.562 \\
\hline Model 3 & 1 (reference) & $0.80(0.61,1.05)$ & $0.94(0.72,1.22)$ & $0.99(0.76,1.29)$ & 0.941 \\
\hline High blood pressure & $86(22.9)$ & $119(31.7)$ & $121(32.1)$ & $150(39.8)$ & \\
\hline Model 1 & 1 (reference) & $1.42(1.07,1.87)$ & $1.46(1.11,1.92)$ & $1.48(1.13,1.93)$ & 0.005 \\
\hline Model 2 & 1 (reference) & $1.41(1.06,1.87)$ & $1.38(1.03,1.84)$ & $1.36(1.03,1.80)$ & 0.046 \\
\hline Model 3 & 1 (reference) & $1.35(1.02,1.79)$ & $1.31(0.99,1.73)$ & $1.22(0.92,1.60)$ & 0.290 \\
\hline Elevated FPG & $92(24.5)$ & $94(25.1)$ & $93(24.7)$ & $114(30.2)$ & \\
\hline Model 1 & 1 (reference) & $1.04(0.73,1.33)$ & $1.05(0.78,1.39)$ & $1.13(0.86,1.49)$ & 0.223 \\
\hline Model 2 & 1 (reference) & $0.99(0.73,1.33)$ & $1.07(0.79,1.44)$ & $0.98(0.73,1.32)$ & 0.658 \\
\hline Model 3 & 1 (reference) & $0.96(0.72,1.29)$ & $1.03(0.77,1.38)$ & $0.99(0.74,1.34)$ & 0.583 \\
\hline
\end{tabular}

Tests for trend were conducted using multivariate Cox proportional hazards models. Values are presented as number (\%) or $\mathrm{HR}(95 \% \mathrm{Cl})$. Model 1: adjusted for age. Model 2: adjusted for marital status, region, education, current smoker, alcohol consumption, hypertension, hyperlipidaemia, diabetes based on Model 1. Model 3: adjusted for WC based on Model 2.

FPG, fasting plasma glucose; HDL-C, high-density lipoprotein cholesterol; MetS, metabolic syndrome; TG, triglyceride; WC, waist circumstance.

tumor necrosis factor (TNF)-alpha. ${ }^{31}{ }^{32}$ A series of inflammatory markers are involved in the maintenance of metabolic homeostasis. For example, inhibition of receptor activator of Nuclear Factor-K B ligand (RANKL) could play a positive role in muscle strength and insulin sensitivity, particularly in the condition of sarcopenia. ${ }^{33}$ IL-15, expressed in skeletal muscle, is involved in the regulation of adipose tissue mass. ${ }^{34}$ In addition, our study found that sex differences existed in the association between relative HGS and MetS. The physiological differences by sex may explain this phenomenon. It has been accepted that the level of testosterone, a hormone in humans, tends to have a strong influence on muscle mass and body fat composition in men. ${ }^{35}$ Declines in testosterone with age could contribute to an unfavourable metabolic profile, including an increased fat mass (resulting in abdominal obesity) and decreased insulin sensitivity. ${ }^{36}$ Oestrogen has a positive effect on receptor-specific activity that promotes glucose transport into the muscle, regulation of myofiber size, and lipid uptake and metabolism. ${ }^{37}$

The strength of this study is that it is a representative nationwide sample of Chinese adults with a relatively long follow-up period. In our coordinated analysis, we controlled for as many potential confounding factors as possible, including sociodemographic factors, lifestyle factors and a history of diseases. However, several limitations of this study should be mentioned. First, although we have considered many confounding factors, other potential variables are likely to exist, such as dietary habits, physical activity, muscle mass and hormone levels. 
Second, information about the serum insulin level was unavailable, and thus, we cannot exclude the possibility that there is a mediating effect of insulin resistance on the association between relative HGS and MetS. Third, the results of the longitudinal analysis could be applicable to ethnic Chinese but not other populations. Finally, the observed associations might not be causal, and therefore, the causality of a pathway from low HGS to MetS should be further explored.

\section{CONCLUSIONS}

In conclusion, relative HGS is inversely and independently associated with the risk of MetS. It was also inversely associated with an increased risk of abdominal obesity, and this association was more pronounced in men than in women. Moreover, the present study provides support for the utility of relative HGS to assess physical condition. Thus, in the future, relative HGS may become a widely used, simple measurement to evaluate muscle strength in the general population in clinical practice.

Acknowledgements The authors appreciate all the participants and we are deeply grateful to the China Health and Retirement Longitudinal Study (CHARLS) for providing the data.

Contributors CS, JL and YX designed the study and drafted the manuscript. YY and ZX participated in revising the manuscript. All authors approved the final manuscript.

Funding This study was supported by Medical Health Science and Technology program of Zhejiang Province (2018KY492 and 2020KY165).

Competing interests None declared.

Patient and public involvement Patients and/or the public were not involved in the design, or conduct, or reporting, or dissemination plans of this research.

Patient consent for publication Not required.

Ethics approval The data collection was obtained from the Biomedical Ethics Review Committee of Peking University (IRB00001052-11015). The use of China Health and Retirement Longitudinal Study data was obtained from the University of Newcastle's Human Research Ethics Committee (H-2015-0290).

Provenance and peer review Not commissioned; externally peer reviewed.

Data availability statement Data are available in a public, open access repository. All of the CHARLS data will be accessible to researchers around the world at the CHARLS project website (http://charls.pku.edu. cn/).

Open access This is an open access article distributed in accordance with the Creative Commons Attribution Non Commercial (CC BY-NC 4.0) license, which permits others to distribute, remix, adapt, build upon this work non-commercially, and license their derivative works on different terms, provided the original work is properly cited, appropriate credit is given, any changes made indicated, and the use is non-commercial. See: http://creativecommons.org/licenses/by-nc/4.0/.

\section{ORCID iD}

Ying Yang http://orcid.org/0000-0002-1822-9546

\section{REFERENCES}

1 Alberti KGMM, Eckel RH, Grundy SM, et al. Harmonizing the metabolic syndrome: a joint interim statement of the International diabetes Federation Task force on epidemiology and prevention; National heart, lung, and blood Institute; American heart association; world heart Federation; international atherosclerosis Society; and international association for the study of obesity. Circulation 2009;120:1640-5.
$2 \mathrm{He} \mathrm{Y,} \mathrm{Wu} \mathrm{W,} \mathrm{Wu} \mathrm{S,} \mathrm{et} \mathrm{al.} \mathrm{Linking} \mathrm{gut} \mathrm{microbiota,} \mathrm{metabolic} \mathrm{syndrome}$ and economic status based on a population-level analysis. Microbiome 2018;6:6-172.

3 Li R, Li W, Lun Z, et al. Prevalence of metabolic syndrome in mainland China: a meta-analysis of published studies. BMC Public Health 2016;16:16-296.

4 Gu D, Reynolds K, Wu X, et al. Prevalence of the metabolic syndrome and overweight among adults in China. Lancet 2005;365:1398-405.

5 Chen Q, Rong P, Zhu S, et al. Targeting RalGAP $\alpha 1$ in skeletal muscle to simultaneously improve postprandial glucose and lipid control. Sci Adv 2019:5-4116.

6 Tieland M, Trouwborst I, Clark BC. Skeletal muscle performance and ageing. J Cachexia Sarcopenia Muscle 2018;9:3-19.

7 Leong DP, Teo KK, Rangarajan S, et al. Reference ranges of handgrip strength from 125,462 healthy adults in 21 countries: a prospective urban rural epidemiologic (pure) study. $J$ Cachexia Sarcopenia Muscle 2016;7:535-46.

8 Janssen I, Heymsfield SB, Ross R. Low relative skeletal muscle mass (sarcopenia) in older persons is associated with functional impairment and physical disability. J Am Geriatr Soc 2002;50:889-96.

9 Cruz-Jentoft AJ, Bahat G, Bauer J, et al. Sarcopenia: revised European consensus on definition and diagnosis. Age Ageing 2019;48:16-31.

10 McGrath R. Comparing absolute handgrip strength and handgrip strength normalized to body weight in aging adults. Aging Clin Exp Res 2019;31:1851-3.

11 Minneci C, Mello AM, Mossello E, et al. Comparative study of four physical performance measures as predictors of death, incident disability, and falls in unselected older persons: the insufficienza cardiaca negli Anziani Residenti a Dicomano study. J Am Geriatr Soc 2015;63:136-41.

12 Leong DP, Teo KK, Rangarajan S, et al. Prognostic value of grip strength: findings from the prospective urban rural epidemiology (pure) study. Lancet 2015;386:266-73.

13 Lawman HG, Troiano RP, Perna FM, et al. Associations of relative handgrip strength and cardiovascular disease biomarkers in U.S. adults, 2011-2012. Am J Prev Med 2016;50:677-83.

14 Yi DW, Khang AR, Lee HW, et al. Relative handgrip strength as a marker of metabolic syndrome: the Korea National health and nutrition examination survey (KNHANES) VI (2014-2015). Diabetes Metab Syndr Obes 2018;11:227-40.

15 Churilla JR, Summerlin M, Richardson MR, et al. Mean combined relative grip strength and metabolic syndrome: 2011-2014 Nationa health and nutrition examination survey. J Strength Cond Res 2020;34:995-1000.

16 Zhao Y, Hu Y, Smith JP, et al. Cohort profile: the China health and retirement longitudinal study (CHARLS). Int J Epidemiol 2014;43:61-8.

17 Peterson MD, Zhang P, Choksi P, et al. Muscle weakness thresholds for prediction of diabetes in adults. Sports Med 2016;46:619-28.

$18 \mathrm{Wu} \mathrm{H}$, Liu M, Chi VTQ, et al. Handgrip strength is inversely associated with metabolic syndrome and its separate components in middle aged and older adults: a large-scale population-based study. Metabolism 2019;93:61-7.

19 Li D, Guo G, Xia L, et al. Relative handgrip strength is inversely associated with metabolic profile and metabolic disease in the general population in China. Front Physiol 2018:9-59.

20 Ho FKW, Celis-Morales CA, Petermann-Rocha F, et al. The association of grip strength with health outcomes does not differ if grip strength is used in absolute or relative terms: a prospective cohort study. Age Ageing 2019;48:684-91.

21 McGrath R, Vincent BM, Al Snih S, et al. The Association Between Muscle Weakness and Incident Diabetes in Older Mexican Americans. J Am Med Dir Assoc 2017;18:452.e7-452.e12.

22 Yang EJ, Lim S, Lim J-Y, et al. Association between muscle strength and metabolic syndrome in older Korean men and women: the Korean longitudinal study on health and aging. Metabolism 2012;61:317-24.

$23 \mathrm{Ji} \mathrm{C}$, Xia Y, Tong S, et al. Association of handgrip strength with the prevalence of metabolic syndrome in US adults: the National health and nutrition examination survey. Aging 2020;12:7818-29.

24 Keevil VL, Luben R, Dalzell N, et al. Cross-Sectional associations between different measures of obesity and muscle strength in men and women in a British cohort study. J Nutr Health Aging 2015;19:3-11.

25 de Carvalho DHT, Scholes S, Santos JLF, et al. Does abdominal obesity accelerate muscle strength decline in older adults? Evidence from the English longitudinal study of ageing. J Gerontol A Biol Sci Med Sci 2019;74:1105-11.

26 Ramírez-Vélez R, Pérez-Sousa Miguel Ángel, García-Hermoso A, et al. Relative handgrip strength diminishes the negative effects 
of excess adiposity on dependence in older adults: a moderation analysis. J Clin Med 2020;9. doi:10.3390/jcm9041152. [Epub ahead of print: 17 Apr 2020].

27 Guillet C, Boirie Y. Insulin resistance: a contributing factor to age-related muscle mass loss? Diabetes Metab 2005;31 Spec No 2:5S20-5.

28 Baker JF, Mostoufi-Moab S, Long J, et al. Intramuscular fat accumulation and associations with body composition, strength, and physical functioning in patients with rheumatoid arthritis. Arthritis Care Res 2018;70:1727-34.

29 Cheng YJ, Gregg EW, De Rekeneire N, et al. Muscle-strengthening activity and its association with insulin sensitivity. Diabetes Care 2007;30:2264-70.

30 Eckel RH, Grundy SM, Zimmet PZ. The metabolic syndrome. Lancet 2005;365:1415-28.

31 Verghese J, Holtzer R, Oh-Park M, et al. Inflammatory markers and gait speed decline in older adults. J Gerontol A Biol Sci Med Sci 2011;66:1083-9.
32 Schaap LA, Pluijm SMF, Deeg DJH, et al. Higher inflammatory marker levels in older persons: associations with 5 -year change in muscle mass and muscle strength. J Gerontol A Biol Sci Med Sci 2009;64:1183-9.

33 Bonnet N, Bourgoin L, Biver E, et al. Rankl inhibition improves muscle strength and insulin sensitivity and restores bone mass. $J$ Clin Invest 2019;129:3214-23.

34 Nielsen AR, Hojman P, Erikstrup C, et al. Association between interleukin-15 and obesity: interleukin-15 as a potential regulator of fat mass. J Clin Endocrinol Metab 2008:93:4486-93.

35 Kelly DM, Jones TH. Testosterone: a metabolic hormone in health and disease. J Endocrinol 2013;217:R25-45.

36 Goldman AL, Bhasin S, Wu FCW, et al. A reappraisal of testosterone's binding in circulation: physiological and clinical implications. Endocr Rev 2017;38:302-24.

37 Gheller BJF, Riddle ES, Lem MR, et al. Understanding age-related changes in skeletal muscle metabolism: differences between females and males. Annu Rev Nutr 2016;36:129-56. 